\title{
Adhesion of functional layer on polymeric substrates for optoelectronic applications
}

E. Amendola amendola@unina.it

\section{A. Cammarano}

\section{Pezzuto \\ D. Acierno}

\author{
Institute of Composite and Biomedical Materials, National Research Council, Piazzale E. Fermi 1, 80055 \\ Portici (NA), Italy \\ Technological District of Polymer and Composite Materials Engineering and Structures, IMAST \\ S.c.a.r.l, Piazzale E. Fermi 1, 80055 Portici (NA), Italy \\ Institute of Composite and Biomedical Materials, National Research Council, Piazzale E. Fermi 1, 80055 \\ Portici (NA), Italy \\ Department of Engineering and Production of Materials, University of Naples "Federico II", Piazzale \\ Tecchio 80, 80125 Naples, Italy
}

The use of plastic film substrates for organic electronic devices promises to enable new applications, such as flexible displays. Plastic substrates have several distinct advantages, such as ruggedness, robustness, ultra lightness, conformability and impact resistance over glass substrates, which are primarily used in flat panel displays (FPDs) today. However, high transparency, proper surface roughness, low gas permeability and high transparent electrode conductivity of the plastic substrate are required for commercial applications. Polyesters, both amorphous and semicrystalline, are a promising class of commercial polymer for optoelectronic applications.

Surface modification of polyester films was performed via chemical solution determining hydrolysis or oxidation. Hydrolysis was carried out by means of sodium hydroxide solution and oxidation by using standard clean 1 (SC-1) of RCA procedure [1]. For this work we have used commercial polymer films of $100 \mu \mathrm{m}$ in thickness: AryLite ${ }^{\mathrm{TM}}$ [2], supplied by Ferrania Imaging Technologies S.p.A. and characterised by very high glass transition temperature, Mylar'TM (Polyethylene Terephthalate PET) and Teonex ${ }^{\mathrm{TM}}$ (Polyethylene Naphthalate PEN) both supplied by Dupont. Moreover, a bioriented and semicrystalline PET have been used. The aim of this study is modifying the polymer surface to improve the adhesion between organic-inorganic layer. It was found that the $\mathrm{NaOH}$ and $\mathrm{SC}-1$ treatment cause a decrease of contact angles. In the present study we have deposited a thin films of amorphous hydrogenated silicon (a-Si: $\mathrm{H}$ ) and its oxide ( $\mathrm{SiO}_{2}$ ) on a new high temperature polymer substrate, AryLite ${ }^{\mathrm{TM}}$, by plasma enhanced chemical vapour deposition (PECVD) [3], with a radio frequency plasma system. [DOI: 10.2971/jeos.2009.09027]

Keywords: PECVD, surface modification, polymeric substrate, AryLite ${ }^{\mathrm{TM}}$

\section{INTRODUCTION}

Electroluminescent devices based on organic materials are of considerable interest owing to their attractive characteristics and potential applications to optoelectronic devices that are electrical-to-optical or optical-to-electrical transducers. Organic light-emitting diodes (OLEDs) have attracted much attention because of their advantages, such as self emission, low weight and a wide viewing angle. Rigid glass substrates are traditionally used for OLED application. However, they are unsuitable for certain applications in modern OLEDs because glass is very brittle, cannot be deformed and is heavy especially for large area display. It's possible to produce an OLED by using a polymer substrate that is flexible, lightweight and robust.

The adoption of polymers for electronic applications has been slowed down due to their limited compatibility with semiconductor fabrication processes. In particular, low glass transition temperature ( $\mathrm{Tg}$ ) of most polymers limit their use to temperatures below $250^{\circ} \mathrm{C}$. Since commercially available polymers do not exhibit all these characteristics, the future display market requires a new generation of plastic materials. Several high-Tg polymers with optical transparency, good chemical resistance and barrier properties have recently been developed for application in organic (OLED) display technology, and these latest developments have motivated the present research.

The advantages by using organic substrates are: low production cost, easy processing, reduction of weight (30\%) and of thickness (50\%) whit respect to glass, flexibility and mechanical robustness. Therefore, the detailed knowledge of thermal properties, mechanical response of substrate single layer and of the adhesion strength between the polymer/inorganic film are of significant importance. The modification of polymer surfaces is a research field that has received much attention because specific surface characteristics are needed in a number of applications, such as adhesion, biomaterials, protective coatings and organic optoelectronic devices.

Commercial polymer films of $100 \mu \mathrm{m}$ in thickness have been used: AryLite ${ }^{\mathrm{TM}}$ [2], supplied by Ferrania Imaging Technologies S.p.A., Mylar ${ }^{\mathrm{TM}}$ (Polyethylene Terephthalate PET) and Teonex ${ }^{\mathrm{TM}}$ (Polyethylene Naphthalate PEN) both supplied by 
Dupont and a bioriented and semicrystalline PET. An amorphous hydrogenated silicon [4] (a-Si:H) and $\mathrm{SiO}_{2}$ functional films have been deposited by plasma enhanced chemical vapour deposition (PECVD) [3] on AryLite ${ }^{\mathrm{TM}}$. The a-Si:H layer reveals the presence of uniformly distributed fractures on the whole surface.

The surface of selected polymers (aromatic polyesters) has been modified in order to improve the adhesion between polymeric substrates and inorganic film and to reduce the inorganic layer cracking surface of selected polymers.

For this study we have used traditional characterization techniques such as Differential Scanning Calorimetry (DSC), Thermogravimetric Analysis (TGA), to evaluate thermal behaviour of plastic substrate, and small volume testing methods such as Micro-Scratch for nanoscale characterization of mechanical and adhesion properties of thin functional films. The modified films were characterized also by water contact angle, optical microscopy and T-Peel adhesion test.

\section{MATERIALS AND METHODS}

\subsection{Materials}

Commercial polymer films of $100 \mu \mathrm{m}$ in thickness have been selected: AryLite ${ }^{\mathrm{TM}}$ (supplied by Ferrania Imaging Technologies S.p.A.) [2] characterised by very high glass transition temperature, Mylar ${ }^{\mathrm{TM}}$, Teonex ${ }^{\mathrm{TM}}$ (PET and PEN supplied by Dupont) and commercial PET.

T-Peel test has been carried out gluing the samples with a Polyurethane thermoset composed by Polurene FP38B and Polurene FP38CS1 used as hardener agent, both supplied by S.A.P.I.C.I. S.p.A.

\subsection{Thermo-mechanical properties of substrates}

Thermal properties of substrates under investigation have been evaluated in order to determine glass transition temperature Tg and degradation temperature by DSC and TGA measurements respectively

The DSC thermal analysis technique measures heat flows and phase changes on a sample under thermal cycles. Since the $\mathrm{Tg}$ is overlaid by an enthalpic relaxation phenomenon, deeper investigations were performed with Modulated DSC.

MDSC raw data are analysed to split the overall behaviour similar to conventional DSC, in two separated contribution, namely "Reversing" and "Non Reversing". The reversing signal provides information on heat capacity and melting, while the "Non Reversing" signal shows the kinetic process of enthalpic recovery at $\mathrm{Tg}$, cold crystallization and crystal perfection.

The glass transition (Tg) was investigated by DSC-Q1000 (TA Instruments). The degradation temperature was investigated by TA2950 (TA Instruments). Samples were heated from $50^{\circ} \mathrm{C}$ to $400^{\circ} \mathrm{C}$, at heating rate of $10^{\circ} \mathrm{C} / \mathrm{min}$. Deeper investigations were performed with Modulated DSC. Samples were heated from $150^{\circ} \mathrm{C}$ to $400^{\circ} \mathrm{C}$, at heating rate of $2.5^{\circ} \mathrm{C} / \mathrm{min}$., with a modulated temperature amplitude of $0.5^{\circ} \mathrm{C}$ and a period of $60 \mathrm{sec}$. both under a nitrogen flow.

Thermal stability was studied by thermogravimetric analysis (TGA). The weight loss due to the formation of volatile products caused by the degradation at high temperature was monitored as a function of temperature. The heating occurred both under a nitrogen and oxygen flow, from room temperature up to $900^{\circ} \mathrm{C}$ with a heating rate of $10^{\circ} \mathrm{C} / \mathrm{min}$.

Elastic modulus and ultimate properties were investigated according to UNI EN ISO 527-3 on rectangular specimens with $150 \mathrm{~mm}$ length, $25 \mathrm{~mm}$ width and $0.1 \mathrm{~mm}$ thick using a mechanical dynamometer SANS 4023 with a $30 \mathrm{kN}$ loading cell and a traverse speed of $20 \mathrm{~mm} / \mathrm{min}$.

Thermal and mechanical properties of materials are shown in Table 1.

\subsection{Surface treatments}

Polymer films were immersed in an alcohol/water $(1 / 1, \mathrm{v} / \mathrm{v})$ solution for 2 hours to clean the surface and subsequently rinsed with a large amount of distilled water. They were dried under reduced pressure for 12 hours at $25^{\circ} \mathrm{C}$. The films were subsequently immersed in an aqueous solution of $45 \mathrm{~mol} / \mathrm{L}$ $\mathrm{NaOH}$ at $75^{\circ} \mathrm{C}$ for hydrolysis reaction.

It's well know that the hydrolysis reaction produce alcohol functionality via nucleophilic addition of the hydroxide group $(\mathrm{OH})$ to the carbonilic group $(\mathrm{C}=\mathrm{O})$ [5] (Figure 1).
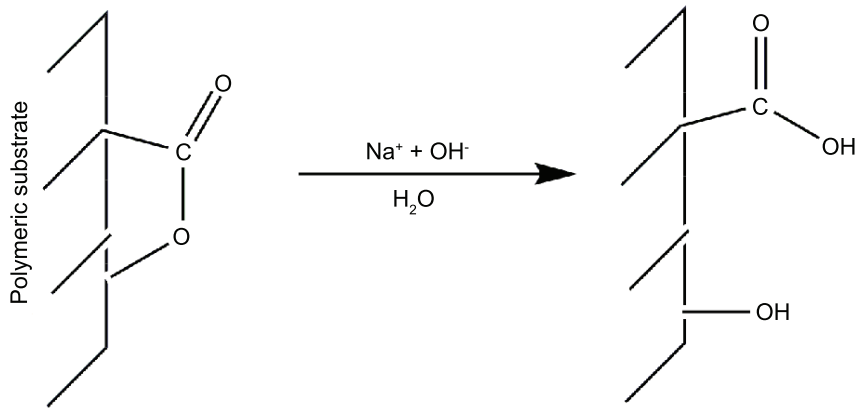

FIG. 1 Scheme of nucleophilic addition of hydroxide group to the carbonilic group

Standard clean 1 (SC-1) of RCA procedure has been used for oxidation reaction on surface. The samples were immersed in an aqueous solution of hydrogen peroxide $30 \%\left(\mathrm{H}_{2} \mathrm{O}_{2}\right)$ and ammonium hydroxide $27 \%\left(\mathrm{NH}_{4} \mathrm{OH}\right)(5 / 1 / 1 ; \mathrm{v} / \mathrm{v} / \mathrm{v})$ at $70^{\circ} \mathrm{C}$ for a given time.

Lian Wang et al. [6] suppose that part of aromatic rings on polyester surfaces were damaged during the oxidation treatment. Formation of $\mathrm{C}=\mathrm{O}$ groups on the treated polyester surfaces may be due to the ring opening and oxidation of polyester aromatic rings. 
After both treatments the films were rinsed with distilled water and then dried under reduced pressure at $25^{\circ} \mathrm{C}$ overnight.

\subsection{Contact angle measurements}

The surface wettability was evaluated by contact angle measurements [7, 8] using the sessile drop method considering the shape of the small liquid drop to be a truncated sphere. The sessile drop contact angle was measured by OCA 20 Dataphysics using $1 \mu \mathrm{L}$. Drop water contact angle were used to assess efficiency of surface modification suffered by the polymer films. Each solid sample was measured five times with liquid at room temperature.

\subsection{T-peel test of adhesion}

The T-peel tests of adhesion [9] were carried out on the untreated and treated substrates using SANS 4023 universal testing machine with a load cell of $30 \mathrm{kN}$ and a peel speed of $10 \mathrm{~mm} / \mathrm{min}$. in accordance to ASTM D 1876-01 (rectangular specimens with $100 \mathrm{~mm}$ length, $25 \mathrm{~mm}$ width and $0.1 \mathrm{~mm}$ thick). We have used polyurethane adhesive as probe for testing the effect of the surface free energy of polymeric substrate. Polyurethane (about $100 \mu \mathrm{m}$ in thickness), was applied on both substrates uniformly.

\section{6 optical microscopy}

The morphology of samples was investigated by means of optical microscopy (Olympus BX51P). Samples have been observed in transmitted and reflected light with a magnification of $20 \times$.

\subsection{Plasma Chemical Vapour Deposition (PECVD)}

Thousand nm thick $\mathrm{SiO}_{2}$ layer was deposited by Plasma Enhanced Chemical Vapour Deposition (PECVD) on both faces of the untreated AryLite ${ }^{\mathrm{TM}}$ substrate. The good thermal insulating properties of the inorganic coating greatly enhances the ability of polymer substrate to withstand the extremely high thermal burst occurring during laser treatments of a-Si:H [4] and provides a smooth surface for the following deposition [3].

Gas pressure and RF power are kept constant at 600 mTorr and $5 \mathrm{~W}$, respectively. Hundred nm thick hydrogenated amorphous silicon (a-Si:H) film was subsequently deposited by PECVD on the $\mathrm{SiO}_{2}$ layer (only on top face) at 750 mTorr and $3 \mathrm{~W}$ using pure $\mathrm{SiH}_{4}$. The film growth rate is approximately of $1.4 \AA / \mathrm{sec}$.

The process temperature, for both $\mathrm{SiO}_{2}\left(120^{\circ} \mathrm{C}-250^{\circ} \mathrm{C}\right)$ and a-Si:H (about $400^{\circ} \mathrm{C}$ ), was determined in accordance to the glass transition temperature and the thermal expansion coefficient of the substrate. The deposition process was performed by Multichamber System MC5000 (ENEA-Portici), a Ultra High Vacuum Multichamber for Plasma Enhanced Chemical Vapour Deposition.

\subsection{Nano-scratch test of thin inorganic layers on AryLite ${ }^{\mathrm{TM}}$ substrates}

In nano-scratch studies, a conical indenter was drawn over the sample surface with a ramping up of the load until damage occurred; the load corresponding to damage provides a measure of scratch resistance or adhesive strength of a coating and is called "critical load". The definition of damage can be the onset of cracking around the scratching tip, spalling of the coating, or the formation of a channel in which all of the coating has been removed from the substrate.

Nano-scratch testing was performed with a Micro Materials NanoTest [10]. The Nanotest is a modular system for nano-indentation, nano-scratch and nano-impact testing. It is a pendulum-based depth-sensing system, with the sample mounted vertically and the load applied electromagnetically. Electrical current in the coil causes the pendulum to rotate on its frictionless pivot so that the diamond probe penetrates the film surface. Test probe displacement is measured with a parallel plate capacitor achieving sub-nanometer resolution. Transverse sample stage motion enables nano-scratch testing, friction, wear and profilometry to be performed as required. A spheroconical diamond probe with nominally $3 \mu \mathrm{m}$ end radius was used for the nano-scratch testing. The experiments involved three sequential scans over the same $250 \mu \mathrm{m}$ track, all at $2 \mu \mathrm{m} / \mathrm{sec}$. scan speed. In the first topography scan the applied load was constant at $0.10 \mathrm{mN}$. Surface roughness was measured from this scan. In the second (scratch) scan, the load applied after $50 \mu \mathrm{m}$ was ramped at a constant rate of $0.1 \mathrm{mN} / \mathrm{sec}$. to a maximum load value of $10.00 \mathrm{mN}$. In the final scan the resultant topografy was observed by using a low applied load of $0.10 \mathrm{mN}$. Five repeat tests were performed on each film. The NanoTest optical microscope and digital capture system was used to image the residual scratch tracks.

\section{RESULTS AND DISCUSSION}

\subsection{Thermo-mechanical properties of substrates}

The use of polymer substrates in microelectronic applications implies issues as: optical, thermal, mechanical and morphological properties, barrier like properties, a good wettability and a high compatibility with different materials that will be deposited. Polymer substrates investigated for possible use in microelectronic applications are amorphous (AryLite ${ }^{\mathrm{TM}}$ ) and semicrystalline thermoplastic (Teonex ${ }^{\mathrm{TM}}$, Mylar $^{\mathrm{TM}}$ and PET)

Thermal and mechanical characterization results are reported in Table 1.

Comparison of the thermal and mechanical behavior of analyzed substrates does not allow to identify a unique best candidate for optoelectronic application. Phase transition temperatures evidence the good performance of amorphous AryLite $^{\mathrm{TM}}$, featuring the highest $\mathrm{Tg}\left(324^{\circ} \mathrm{C}\right)$ and good optical transparency. The lack of crystalline phase is a consequence of the aromatic nature of polymer backbone which hinders conformational rearrangements into a regular crystalline structure. On the other hand the rigid backbone is responsible 


\begin{tabular}{|l|c|c|c|c|}
\hline & AryLite & Mylar & TM & Teonex \\
\hline Glass transition temperature $T_{g}\left[{ }^{\circ} \mathrm{C}\right]$ & 324 & 80 & 122 & 82 \\
\hline Melting temperature $T_{m}\left[{ }^{\circ} \mathrm{C}\right]$ & - & 250 & 153 & 260 \\
\hline Initial degradation temperature $\left[{ }^{\circ} \mathrm{C}\right]$ & 488 & 410 & 398 & 405 \\
\hline Young's Modulus $E_{s}[\mathrm{GPa}]$ & 2.4 & 4.5 & 6.3 & $3.2^{*}$ \\
\hline Yield strenght $\sigma_{y}[\mathrm{MPa}]$ & - & 102 & 153 & $108^{*}$ \\
\hline Elongation at break $\varepsilon_{r}[\%]$ & 10 & 72 & 31 & $40^{*}$ \\
\hline
\end{tabular}

TABLE 1 Thermo-Mechanical properties of Arylite ${ }^{\mathrm{TM}}$, Teonex ${ }^{\mathrm{TM}}$, Mylar ${ }^{\mathrm{TM}}$ and PET. *Average value between parallel and perpendicular direction

of reduced elongation at break and the lack of crystalline reinforcement results in poor elastic and ultimate properties.

Nevertheless, a balanced compromise of overall properties has determined the selection of AryLite ${ }^{\mathrm{TM}}$ between the investigated materials.

Since the $\mathrm{Tg}$ is overlaid by an enthalpic relaxation phenomenon, deeper investigations were performed with Modulated DSC. Samples were heated from $150^{\circ} \mathrm{C}$ to $400^{\circ} \mathrm{C}$, at heating rate of $2.5^{\circ} \mathrm{C} / \mathrm{min}$., with a modulated temperature amplitude of $0.5^{\circ} \mathrm{C}$ and a period of $60 \mathrm{sec}$. both under nitrogen flow.

Reversing Heat Flow signal is cleansed by influence of enthalpy phenomenon, which affects only Non Reversing Heat Flow signal, and reveals a glass transition temperature ( $\mathrm{Tg}$ ) of $324^{\circ} \mathrm{C}$ (Figure 2).

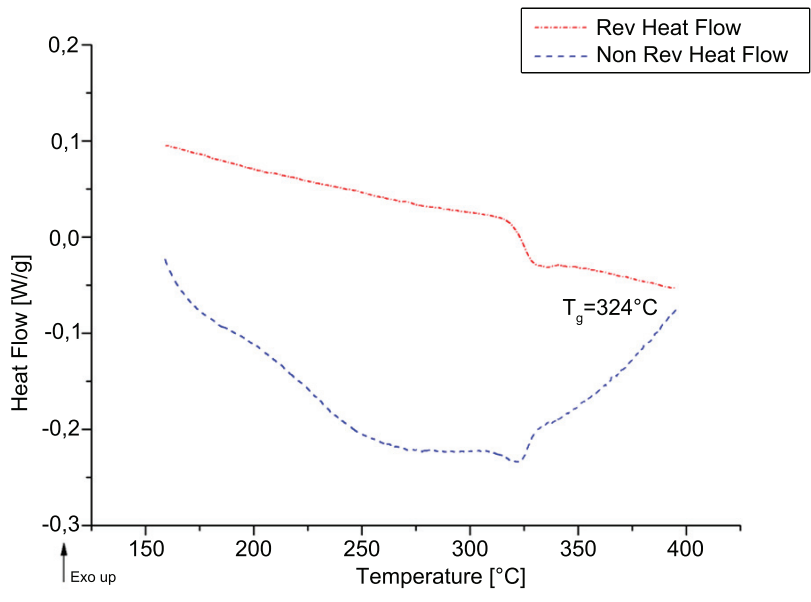

FIG. 2 Modulated Differential Scanning Calorimetry of AryLite ${ }^{\mathrm{TM}}$

\subsection{Optical morphology and nano-scratch test of thin inorganic layers of AryLite ${ }^{\mathrm{TM}}$}

The morphology and micromechanical analysis and nanoscratch tests of samples was investigated by means of optical microscopy.

Both sides of sample of AryLite ${ }^{\mathrm{TM}}$ has been observed after deposition to investigate the uniformity of $\mathrm{SiO}_{2}$ film and a-Si:H deposited (Figure 3).

The a-Si:H and $\mathrm{SiO}_{2}$ layers reveal the presence of uniformly distributed fractures on the whole surface. The effect can be

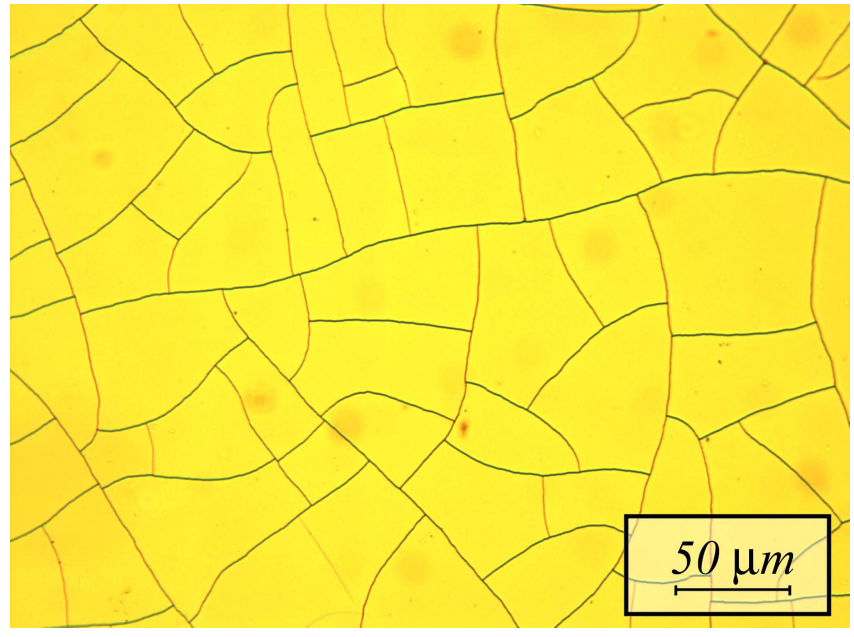

FIG. 3 Optical microscopy of a-Si:H film deposited as top layer. Magnification of $20 \times$

attributed to a mismatch of mechanical properties and coefficient of thermal expansion (CTE) between the polymeric substrate and the inorganic rigid films.

The average dimension of polygonal areas is about 50 microns, with fracture directions distributed isotropically along the sample surface.

The presence of surface fractures and cracks can be attributed to a poor adhesion between the substrate and the inorganic films.

Micromechanical tests on amorphous hydrogenated silicon and on $\mathrm{SiO}_{2}$ layers deposited on AryLite ${ }^{\mathrm{TM}}$ have been carried out to further investigate the interface between organic substrate and inorganic layers.

The load corresponding to the damage provides a measure of scratch resistance or adhesive strength of a coating and is called "critical load" (Lc).

The Lc value is $4.2 \pm 0.1 \mathrm{mN}$ and it is similar with and without a-Si:H layer (Figure 4); this suggests that the dramatic failure of multilayered composite occurred between $\mathrm{SiO}_{2}$ and polymer.

Critical failure load is similar on both sides. Nanoscratch data exhibited a very clear and reproducible critical load at $4.2 \mathrm{mN}$ when a $3 \mu \mathrm{m}$ indenter was used. The failure of the multilayer structure involves blistering and delamination consistent with a brittle coating. 


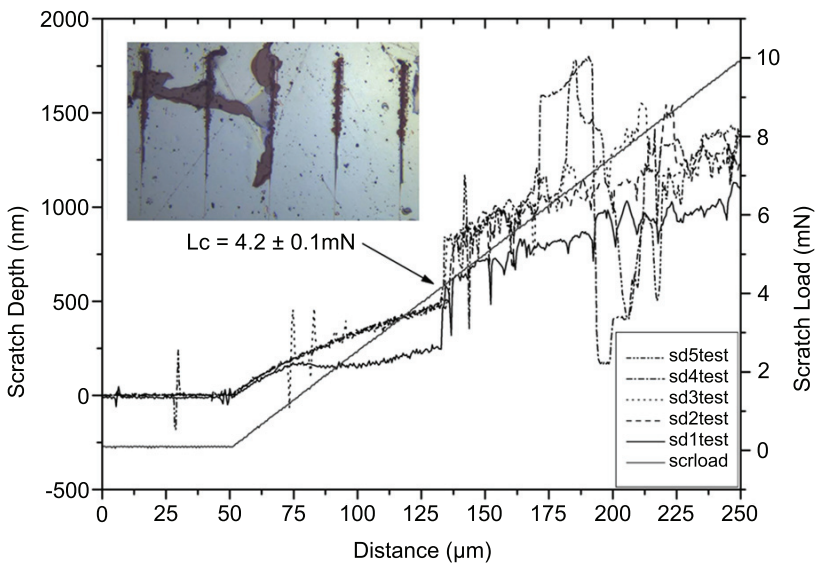

FIG. 4 Comparison of Scratch tests curves on a-Si:H side (with optical image in the plot inset)

The polymer surface of AryLite ${ }^{\mathrm{TM}}$ has been modified to improve the adhesion between organic-inorganic layer and to reduce the inorganic layer cracking surface.

\subsection{Contact angle (CA)}

Figure 5 shows the CA changes for untreated and modified polymer films with $\mathrm{NaOH}$ solution for a given time.

Teonex $^{\mathrm{TM}}$ and Mylar ${ }^{\mathrm{TM}}$ films exhibit a similar behaviour after exposure for 60 minutes at $75^{\circ} \mathrm{C}$ in $\mathrm{NaOH}$, with a decrease in CA solution from $84^{\circ}$ to $44^{\circ}$ and from $81^{\circ}$ to $44^{\circ}$ respectively. PET shows an even larger decrease in water contact angle from $80^{\circ}$ to $35^{\circ}$, while the same treatment induces a smaller effect for AryLite ${ }^{\mathrm{TM}}$, that shows a reduction in the CA from $92^{\circ}$ to $82^{\circ}$. NaOH treatment longer than 30 minutes resulted in severe damage of substrate surface, with reduction of gloss and optical transparency for all samples.

Polymer substrates have been exposed also to oxidation procedure (SC-1) [10] RCA. The solution RCA SC-1 is formed by 5 volumes water $\left(\mathrm{H}_{2} \mathrm{O}\right), 1$ volume $27 \%$ ammonium hydroxide $\left(\mathrm{NH}_{4} \mathrm{OH}\right), 1$ volume $30 \%$ hydrogen peroxide $\left(\mathrm{H}_{2} \mathrm{O}_{2}\right)$ at the

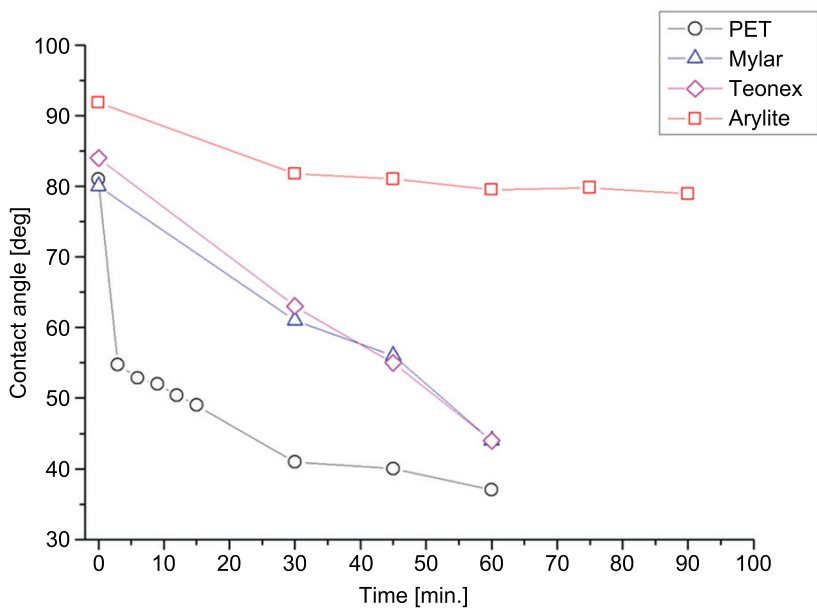

FIG. 5 Water Contact Angle of PET, Mylar ${ }^{\mathrm{TM}}$, Teonex ${ }^{\mathrm{TM}}$ and AryLite ${ }^{\mathrm{TM}}$ treated with $\mathrm{NaOH}$ solution at different time.

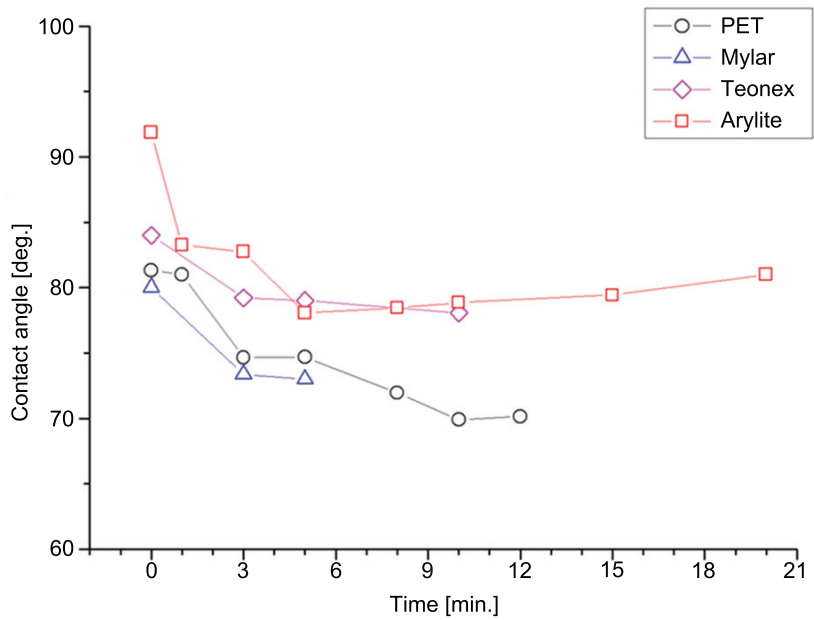

FIG. 6 Water Contact Angle of PET, Mylar ${ }^{\mathrm{TM}}$, Teonex ${ }^{\mathrm{TM}}$ and AryLite ${ }^{\mathrm{TM}}$ treated with SC-1 solution at different time.

operating temperature of $70^{\circ} \mathrm{C}$. A vigorous bubble evolution starts 1-2 minutes after the addition of $\mathrm{H}_{2} \mathrm{O}_{2}$ solution, indicating that the solution is ready for use.

Figure 6 shows a decrease in CA for all materials treated with SC-1 solution in comparison to untreated films.

Lifetime of SC-1 solution is limited to 20 minutes. Therefore, polymer substrates are treated with RCA solution for 15 minutes, when a decrease of gas evolution is observed.

Both methods are effective in increasing the hydrophilicity of samples, but the decrease in CA obtained by SC- 1 treatment is lower than $\mathrm{NaOH}$ solution treatment.

\subsection{T-peel test of adhesion}

For testing the effect of surface free energy of polymeric substrate we have also used T-Peel test of adhesion, using polyurethane adhesive as probe. Table 2 shows the values of T-peel strength $[\mathrm{kN} / \mathrm{m}]$.

It was not possible to measure the Peel strength of AryLite $^{\mathrm{TM}}$ film because it is a brittle material and samples of AryLite ${ }^{\mathrm{TM}}$ break during the test of adhesion.

The T-peel test has confirmed the improved adhesion of polymeric substrates after each treatment. Mylar ${ }^{\mathrm{TM}}$ sample shows the greatest increase of T-Peel strength reaching the value of $1.18 \mathrm{kN} / \mathrm{m}$, twice greater than the untreated one $0.69 \mathrm{kN} / \mathrm{m}$.

\begin{tabular}{|l|c|c|c|c|}
\hline & AryLite $^{\mathrm{TM}}$ & Mylar $^{\mathrm{TM}}$ & Teonex $^{\mathrm{TM}}$ & PET \\
\hline Untreated & - & 0,69 & 0,87 & 0,71 \\
\hline $\begin{array}{l}\mathrm{NaOH} \\
\text { Solution }\end{array}$ & - & 1,18 & 1,31 & 1,07 \\
\hline $\begin{array}{l}\text { SC-1 } \\
\text { Solution }^{b}\end{array}$ & - & 0,75 & 1,10 & 0,81 \\
\hline
\end{tabular}

TABLE 2 T-Peel strength $[\mathrm{kN} / \mathrm{m}]$ of AryLite ${ }^{\mathrm{TM}}$, Teonex ${ }^{\mathrm{TM}}$, MylarTM and PET. ${ }^{a}$ treatment of 30 minutes, ${ }^{b}$ treatment of 5 minutes 


\section{CONCLUSION}

In the present study $\mathrm{SiO}_{2}$ and amorphous hydrogenated silicon (a-Si:H) have been deposited on untreated AryLite ${ }^{\mathrm{TM}}$ substrate by plasma enhanced chemical vapour deposition (PECVD). Small volume testing method such as NanoScratch, has been used for characterize interfaces of multilayer composite.

The presence of surface fractures and cracks is a clear evidence of a poor adhesion at the interface between substrate and inorganic films. Substrates have been treated with $\mathrm{NaOH}$ solution and SC-1 solution to enhance the adhesion characteristic. In both cases a significant decrease of water contact angle have been measured for treated samples resulting in increased wettability of polymeric substrates. Consequently, an increase of T-peel adhesion has confirmed the improved adhesive strength of polymeric substrates after solution treatments. The suitable experimental conditions were found to be $75^{\circ} \mathrm{C}$ and 30 minutes for $4.5 \mathrm{M} \mathrm{NaOH}$ solution. Under this condition the surfaces have the lowest contact angle and no significant damage of the optical or structural properties have been detected. It was found that also the SC-1 treatment caused a decrease of contact angles and an increase of surface energy leading to the improved surface hydrophilicity.

T-peel test has confirmed the improved adhesion of polymeric substrates after treatments.

Moreover T-peel results obtained using polyurethane adhesive lead to foresee the possibility to use a coupling agent (organosilicon monomers) with isocyanate functionality suitable to graft on the organic substrate.

Future works will be focused on the deposition of $\mathrm{SiO}_{2}$ and a-Si:H on treated AryLite ${ }^{\mathrm{TM}}$ substrate. Nano-scratch tests will be carried out in order to study the adhesion at the interface between substrate and inorganic films.

\section{ACKNOWLEDGEMENTS}

The authors gratefully acknowledge Enea of Portici for PECVD on polymeric substrates. The activities where per- formed in the framework of FIRB projects "Micropolys" (RBNEO17JZF) funded by the Ministero dell' Istruzione, dell' Universitá e della Ricerca (MIUR) and "Poliflex" (RBIP06SH3W) granted to IMAST S.c.a.r.l. We also would like to acknowledge Ferrania S.p.A. for providing AryLite ${ }^{\mathrm{TM}}$ substrates.

\section{References}

[1] C. Bogdan, E. Donose, E. Taran, I. U. Vakarelski, H. Shinto, and K. Higashitani "Effects of cleaning procedures of silica wafers on their friction characteristics" J. Colloid Interf. Sci. 299, 233-237 (2006).

[2] S. Angiolini and M. Avidano P-27: Polyarylite Films for Optical Applications with Improved UV-Resistance (Organic Synthesis Lab. Polymers, Ferrania Imaging Technologies, Technical Information).

[3] A. Imparato, C. Minarini, A. Rubino, P. Tassini, F. Villani, A. Guerra, E. Amendola, and D. Della Sala "Thin silicon films on polimeric substrates" Macromol. Symp. 228, 167-176 (2005).

[4] A. Imparato, C. Minarini, A. Rubino, P. Tassini, F. Villani, A. Guerra, E. Amendola, D. Della Sala, M. Kunst, H. C. Neitzert, and S. Bellone "Excimer laser induced crystallization of amorphous silicon on flexible polymer substrates" Thin Solid Films 487, 58-62 (2005).

[5] J. M. Goddard and J. H. Hotchkiss "Polymer surface modification for the attachment of bioactive compounds" Prog. Polym. Sci. 32, 698-725 (2007).

[6] L. Wang, L. Yan, P. Zhao, Y. Torimoto, M. Sadakata, and Q. Li "Surface modification of polystyrene with atomic oxygen radical anions-dissolved solution" Appl. Surf. Sci. 254, 4191-4200 (2008).

[7] G. Mack "Determination of contact angles from measurements of the dimension of small bubbles and drops. I. The spheroidal segment methods for acute angles" J. Phys. Chem. 40, 159-167 (1936).

[8] C. Ozcan and N. Hasirci "Evaluation of Surface Free Energy for PMMA Films" J. Appl. Polym. Sci. 108, 438-446 (2008).

[9] S. D. Desai, A. L. Emanuel, and V. K. Sinha "Polyester Polyol-Based Polyurethane Adhesive; Effect of Treatment on Rubber Surface" J. Polym. Res. 10, 141-149 (2003).

[10] B. D. Beake and S. P. Lau "Nanotribological and nanomechanical properties of 5-80 nm tetrahedral amorphous carbon films on silicon" Diam. Relat. Mater. 14, 1535-1542 (2005). 